\title{
Calculation of the Neutrino Mass
}

\author{
Dr. Andreas Gimsa \\ Stirling Technologie Institut gemeinnützige GmbH, D-14478 Potsdam, Germany
}

\begin{abstract}
The determination of the neutrino mass is considered an important milestone in physics and especially in cosmology. Because it is so extraordinarily small, the usual methods for determining the mass of elementary particles fail. After Wolfgang Pauli's prediction of the neutrino in 1930, experimental proof was not possible until 1956, when an electron antineutrino met a proton and produced a positron and a neutron. The Karlsruhe Tritium Neutrino Experiment KATRIN [1.] is intended to determine the neutrino mass with unique accuracy or, if the sensitivity of the measuring technique is not yet sufficient, to further limit its upper limit. A theoretically exact determination of mass is not yet possible. The present publication is dedicated to this topic. Assuming a mass decay in the universe that includes the neutrino mass, a precise calculation method is proposed and subsequently justified. The effects of neutrino splitting from the proton are examined. In a cosmological perspective, further effects that neutrino decay could have on the expansion of space, gravity, dark mass, magnetic monopoles and time are investigated.
\end{abstract}

Keywords: Neutrino Mass, Proton Decay, Expansion of Space, Gravity, Dark Mass, Magnetic Monopoles, Time.

\section{Introduction}

In nature, a universal mass decay takes place. With this knowledge, some important questions of cosmology can be answered. The universe is an enclosed system. There can be no transport of energy and matter across the system boundaries. The expansion of space takes place isentropically, i.e. with constant entropy. The number of effects in the universe must remain constant. The following formula applies to the entropy of the universe [2.]:

$$
S_{\text {uni }}=\frac{\mathrm{k}}{\hbar} m_{\text {uni }} t_{\text {uni }} c^{2}=\frac{\mathrm{k}}{\hbar} N \hbar=N k
$$

$N$ Has the value of $6.510^{121}$ effects. This can be interpreted as potential information, or as the number of microstates of the universe. So the mass $m_{\text {uni }}$ of the universe must fall with its age $t_{\text {uni }}$. With it the masses of all celestial bodies with any mass $m_{x}$ fall. The following applies: $m_{x} t_{\text {uni }}=$ const. The mass decay of each mass with time can be written as follows:

$$
m_{b}=\frac{m_{a}}{1+\frac{\Delta t}{t_{\text {uni } a}}}
$$

Since the mass of space and all object masses are predominantly related to the proton mass, the mass decay must be proton decay.

If a proton loses a neutrino within a certain time, its basic structure could remain intact. Since the electric charge is conservation variable, the decay increases the mass-related charge.

The assumption that proton decay could occur in the form of a neutrino loss if its basic structure is retained results from the consideration that there must be a subsequent neutrino decay and an associated time formation process.

Neutrinos are neutral particles. Their neutrality can be attributed to a balanced magnetic charge. In general, physical neutrality is based on balanced charges. An electron neutrino could consist of a magnetic north and a magnetic south pole. If an electron neutrino decays, both monopoles are released and they probably form time. This would mean that electron neutrinos would decay all over space. Time, which has always been taken for granted in physical processes, must have a physical cause. It is highly probable that time is created 
only with the neutrino decay and appears as a stream of magnetic monopoles. The monopoles could not be found in space for exactly this reason. This would complete the Maxwell equations: There are sources of magnetic charges in nature, but they are not located in space but in time.

In connection with the calculation of the neutrino mass, the phenomena proton decay, neutrino decay and time origin are physically investigated.

\section{Calculation of the neutrino mass}

To check whether a proton can lose an electron neutrino, it would be valuable to know the exact value of this neutrino mass. For this purpose, corresponding calculation rules are given here for the first time.

All found calculation rules refer to the hydrogen atom in the ground state. The equations captivate by their simplicity and lead all to the same result, an electron neutrino mass of $m_{v}=3.5410^{-37} \mathrm{~kg}$. This corresponds quite exactly to an energy equivalent of $0.2 \mathrm{eV}$. The Karlsruhe neutrino experiment KATRIN is just about to work with this sensitivity [1.]. This means that a proof should just be possible. Equation (12) is derived and the value found there and other known physical correlations prove that equations (03) to (11) must also be correct.

Equation (03) shows the simplest form of calculation. It includes the effective quantum $\mathrm{h}$ and the common period $\mathrm{T}$ of electron and proton:

$$
m_{v}=\frac{\alpha \hbar}{t c^{2}}=\frac{\alpha h}{T c^{2}}
$$

Equation (04) uses the Rydberg wavelength $\lambda_{r y d}$ or the Rydberg frequency $f_{r y d}$ instead of the period:

$$
m_{v}=\frac{4 \pi \alpha \hbar}{\lambda_{r y d} c}=\frac{4 \pi \alpha \hbar}{c^{2}} f_{r y d}
$$

A special notation with the Rydberg energy shows equation (05):

$$
m_{v}=\frac{2 \alpha}{c^{2}} E_{r y d}
$$

Equation (06) reveals a charge-related notation with the electric and magnetic elementary charge $e, p$ and the Rydberg frequency $f_{r y d}$ :

$$
m_{v}=\frac{e p}{\lambda_{r y d} c}=\frac{e p}{c^{2}} f_{r y d}
$$

In a similar way, the neutrino mass can be formulated with the Rydberg constant:

$$
m_{v}=\frac{e p}{4 \pi t c^{2}}=\frac{e p}{c} R_{\infty}
$$

Equation (08) shows the close relationship between neutrino mass and fine structure constant and the masses of electron and proton:

$$
m_{v}=\alpha^{3} \frac{m_{e} m_{p}}{m_{e}+m_{p}} \approx \alpha^{3} m_{e}
$$

Equation (09) shows a similar form and, like equation (03), includes the reduced period $t$ and the centre of gravity distance $r_{s p}$ :

$$
m_{v}=\frac{\hbar^{2}}{r_{s p} t c^{3}} \frac{m_{e}+m_{p}}{m_{e} m_{p}}
$$

Equation (10) establishes a relationship between the neutrino mass and the kinetic energy of the electron and proton of the hydrogen atom in the ground state:

$$
m_{v}=\frac{\alpha}{c^{2}}\left(m_{e} v_{e}{ }^{2}+m_{p} v_{p}{ }^{2}\right)=\frac{2 \alpha}{c^{2}}\left(E_{\text {kin } e}+E_{\text {kin } p}\right)
$$

Equations (11) and (12) are similar and show a relationship between the neutrino mass and two identical electric or magnetic charges:

$$
m_{v}=\frac{1}{c^{2}} \frac{e^{2}}{e_{0} \lambda_{r y d}}=\frac{1}{c^{2}} \frac{p^{2}}{\mu_{0} \lambda_{r y d}}
$$




$$
m_{v}=\frac{\alpha}{c^{2}} \frac{e^{2}}{4 \pi \varepsilon_{0} r_{s p}}=\frac{\alpha}{c^{2}} \frac{p^{2}}{4 \pi \mu_{0} r_{s p}}
$$

According to equation (01) can be written for the entropy of the neutrino:

$$
S_{v}=\frac{\mathrm{k}}{\hbar} m_{v} t c^{2}
$$

where $t$ is the reduced period in the hydrogen atom. With equation (03) a very interesting and simple relation follows:

$$
S_{v}=\alpha k
$$

The following relationship, which results from the relationship between the micro- and macrostructure of universe, can be used for the calculation of the neutrino mass [4.]:

$$
\frac{m_{u n i}}{m_{p}} \frac{r_{u n i}}{r_{p}}=\frac{s_{u n i}}{4 k}
$$

With equations (01), (13), (14) and (15) then follows for the neutrino mass:

$$
m_{v}=\frac{\alpha m_{p} r_{p}}{4 c t}
$$

Like (08), another formula for calculating the neutrino mass includes the masses of proton and electron:

$$
m_{v}=\frac{5}{12} \pi \frac{m_{e}^{3}}{m_{p}{ }^{2}}
$$

The proof for formula (17) can be provided by (16), the context $4 \hbar=c m_{p} r_{p}$ and the calculation rule for the fine structure constant $\alpha$ [4.]:

$$
\alpha=\frac{\pi^{2}}{6} \frac{3}{4} \frac{1}{13^{2}} \frac{m_{p}}{m_{e}+m_{p}}
$$

If the fine structure constant is known, then the mass of the proton can be used to calculate the mass of the electron:

$$
m_{e}=m_{p}\left(\frac{1}{\alpha} \frac{\pi^{2}}{6} \frac{3}{4} \frac{1}{13^{2}}-1\right)
$$

\section{Justification of the neutrino mass calculation using equation ....12}

Equation (12) is particularly interesting because it tells us something about the possible neutrino decay. It contains the energy required to separate two elementary magnetic charges, which are in the ground state in the proton on the center of gravity orbit with the radius $r_{s p}$ of the hydrogen atom, from there to infinity. Part of the energy from the strong force becomes available in the process. This means that only the energy that is smaller by the ratio $\alpha=F_{\text {em }} / F_{\text {strong }}$ is required for the charge separation. After the separation of the charges, the strong force therefore only acts on the mass reduced by exactly one neutrino:

$E_{v}=m_{\nu} c^{2}=\alpha \int_{r=r_{s p}}^{\infty} F_{m g} d r=\alpha \frac{p^{2}}{4 \pi \mu_{0}} \int_{r=r_{s p}}^{\infty} \frac{1}{r^{2}} d r=-\left.\alpha \frac{p^{2}}{4 \pi \mu_{0}} \frac{1}{r}\right|_{r=r_{s p}} ^{\infty}=\alpha \frac{p^{2}}{4 \pi \mu_{0} r_{s p}}$

The separation of both charges to infinity could mean transporting them out of space into the two time dimensions (see 4.)

With equation (20), the neutrino mass can be determined immediately if the size of the elementary magnetic charge $p$ is known. A relevant calculation formula has already been published in [3.].

\section{Effects of neutrino splitting on the proton}

How long does it take today for a single proton to become lighter by one neutrino mass? The following equation with $t_{0}$ as space age can be used for this purpose [4.]:

$\Delta t_{m z}=t_{0}\left(\frac{1}{1-\frac{\Delta m_{m z}}{m_{0} p}}-1\right)=4.35210^{17}\left(\frac{1}{1-\frac{3.5410^{-37}}{1.6710^{-27}}}-1\right) s=9.2010^{7} \mathrm{~s} \approx 2.92 \mathrm{a}$

Although a good 3 years is a long time, you have to consider how many protons the objects consist of. Decay therefore takes place everywhere and all the time. 
It is now also interesting to find out by how much the proton radius has grown during this time. With mass decay, the product of mass and radius must remain constant [4.]. In this source the current proton radius $r_{p}$ was also determined exactly. It applies to the radius increase $\Delta r_{p}$ after 2.92 years:

$$
\begin{aligned}
& \Delta r_{p}=r_{b p}-r_{0 p}=r_{0 p}\left(\frac{m_{0 p}}{m_{b p}}-1\right)=8.3910^{-16}\left(\frac{1.6710^{-27}}{1.6710^{-27}-3.5410^{-37}}-1\right) m \\
& \Delta r_{p}=1.7810^{-25} \mathrm{~m}
\end{aligned}
$$

The radius magnification of the proton is small, but not zero. It is astonishing that this radius magnification $\Delta r_{p}$ of the proton corresponds exactly to the neutrino radius $r_{v}$ under a certain condition. This precondition according to equation (23) is similar to a relationship [4.] obtained between proton and electron $\left(m_{e} r_{p}=\right.$ $\left.m_{p} r_{e}\right)$ :

$$
m_{v} r_{e}=m_{e} r_{v} \quad \text { or } \quad \frac{m_{v}}{r_{v}}=\frac{m_{e}}{r_{e}}
$$

The mass-radius ratio of the electron corresponds to that of the electron neutrino.

If numbers are used here, this leads to the astonishing result mentioned above that the proton, in the time it loses the mass of a neutrino, grows by exactly the radius corresponding to a neutrino radius:

$$
r_{v}=\frac{m_{v} r_{e}}{m_{e}}=\frac{3.5410^{-37} 4.5710^{-19}}{9.1110^{-31}} m=1.7810^{-25} \mathrm{~m}
$$

Herein could lie a deep microstructural truth. To a certain extent, when the neutrino leaves the proton, it seems to give it its radius, by which the proton radius then increases. This could be an indication that this is a quantised increase in radius, the smallest change in which corresponds to the neutrino radius.

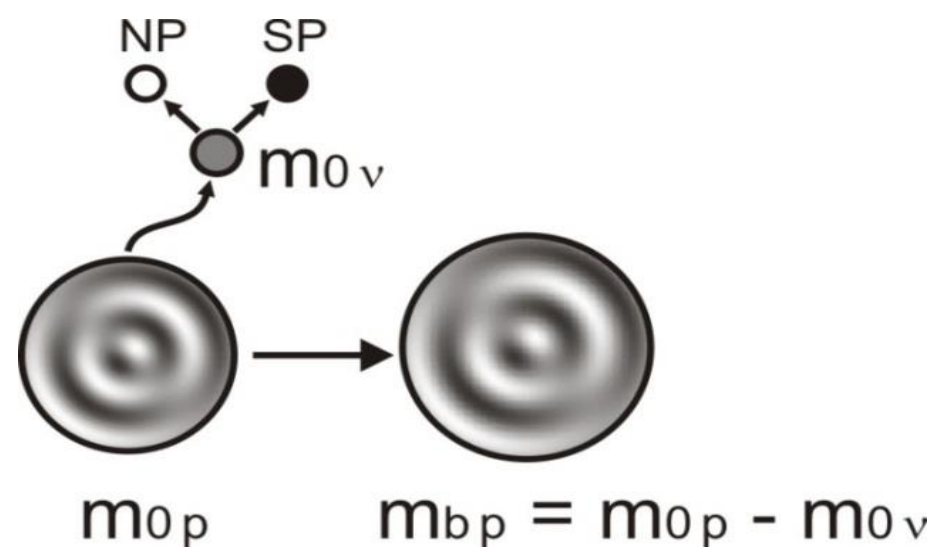

Figure 1: Schematic proton decay at proton magnification and emission of a neutrino, which subsequently decays into two magnetic monopoles

Figure 1 illustrates the presumed proton decay when a neutrino splits off from the proton and the neutrino subsequently decays into a magnetic north pole NP and a magnetic south pole SP, which form time.

The proton becomes slightly larger due to the loss of mass and structure. While the neutrinos are detectable in space in principle, it is assumed that the magnetic monopoles leave space and form time.

\section{Cosmological outlook}

When the neutrinos decay, the mass must become smaller and time must be allowed for because of the required entropy constancy. At the same time, energy conservation applies in the form that with mass decay, the potential gravitational energy that can be released must become less negative. The negative gravitational energy must have a positive mass equivalent for the following reason.

Equal positive masses attract each other and the gravitational field is also attracted by the mass. Because the gravitational field strength $g=\gamma \mathrm{m} / r^{2}$ is greatest near the mass and decreases quadratically with distance.

The gravitational field strength decreases with time in proportion to the mass: The gravitational value $\gamma$ increases quadratically, the square of the distance in the denominator increases to the same extent and the mass becomes proportionally smaller with time [4.]. 
If the gravitational energy is negative with a positive mass equivalent, the negative sign must have a special meaning, a proof of the existence of imaginary light with the velocity $c_{i}$.

For gravitational energy a mass equivalent can be given: $E_{\text {grav }}=m c_{i}{ }^{2}=-m c^{2}$

Imaginary light can only propagate in an imaginary medium. This is given by the imaginary time $t_{i}$, which represents the second time dimension. The existence of imaginary time can be clearly explained: In the imaginary past lies an event that is so far away that the speed of light is not sufficient to influence an event here and now. For example, a solar eruption six minutes ago cannot influence an event here and now. It lies in the imaginary past $t_{i v}$ of the event here and now. In the imaginary future lies an event that is so far away that the speed of light is not sufficient to be influenced by an event here and now. For example, an event on the sun in 6 minutes cannot be influenced by a laser beam emitted here and now in the direction of the sun. The solar event lies in the imaginary future $t_{i z}$ of the emitted laser beam.

The propagation speed $c_{i}$ of imaginary light has no real limit. If it can be proved that the imaginary photons are the gravitational interaction particles, their instantaneous interaction is confirmed [4.].

"Let's look at the Earth and the Sun and assume a gravitational propagation speed that corresponds to the speed of light. Then a force would act on the Earth towards the place where the Sun was eight minutes ago and a force would act on the Sun towards the place where the Earth was eight minutes ago. This delay would mean that the distance between the Sun and the Earth would constantly increase, which means that the orbits would be unstable. The same would be true for Earth and the Moon." [5.]

Dark mass could be present as a negative, repulsive mass. Because masses with the same polarity attract each other, masses with different polarity would have to repel each other.

This would explain the high orbital speeds of stars in the outer regions of galaxies [4]. The stars would be pressed into the interior of the galaxies by dark masses outside. Where does this repulsive mass come from?

After the exit of a magnetic north pole and a south pole from an electron neutrino, the north poles remain near the mass. They appear as gravitation. The south poles are repelled by them, attract each other and reach the outer area of the galaxies. They appear as dark mass [4.]. Both types of monopoles are time formers and each have a real and an imaginary time component. The north poles form the real and the imaginary future, they are attracted by positive mass. The south poles form the real and the imaginary past, they are attracted by negative mass.

The north poles, which exit from the neutrinos during decay, strive into the gravitational field of the mass. Since the field has a positive mass but a negative energy, this energy becomes less negative when the north poles are included. The south poles, which exit from the neutrinos during mass decay, strive towards the outer region of the galaxy. Since the field there has a negative mass but a positive energy, this energy becomes less positive when the north poles are included. Thus, the field energy amounts of the negative attracting and the positive repelling field energies become smaller with the mass decay. It is logical that the field energy must develop proportionally to the mass.

The accelerated expansion of space is caused by the masses of celestial bodies and galaxies that decrease with time. For a given escape velocity of a given mass, it must naturally increase as the mass decreases. This requires the conservation of momentum completely without dark energy.

If time is formed by means of a magnetic monopole current, a magnetic potential must be present. This potential is present between the north poles emerging from the masses and the north poles of the gravitational field, and between the south poles emerging from the masses and the south poles in the outer regions of the galaxies.

In every galaxy, there should be a large repulsive potential between the massive centre and the dark mass.

The neutrino decay of the masses initiates a north pole current into the gravitational field (time flows into the future) and an opposite south pole current into the dark mass (events flow into the past).

$$
t=\frac{d Q_{m g}}{d I_{m g}} \quad Q_{m g}=N p
$$


Equation (25) means that the time flow represents a magnetic charge $Q_{m g}$ which varies with the magnetic current $I_{m g}$. It arises from magnetic monopoles $p$ that flow one-dimensionally and thereby form order. The magnetic charge has the SI unit [Vs]. The magnetic current, like the electric voltage, must have the SI unit [V].

Time is the simplest order system, it runs continuously from the past into the future. Therefore, time must have an information equivalent. The time-information equivalent found by the author $I=t c^{2}$ can also be explained by the loss of structure during mass decay [2.].

\section{Summary}

An exact value for the neutrino mass could be calculated. This value can just about be proven with the Karlsruhe tritium neutrino experiment KATRIN. The calculation of the neutrino mass is based on the assumption that neutrinos can decay. This has an important significance for explaining the expansion of space, gravity, dark mass, magnetic monopoles and time. The calculations for the neutrino mass could be derived from the hydrogen atom. This underlines its fundamental importance.

\section{References}

[1.] Aker, M., Altenmüller, K., Arenz, M. et al. First operation of the KATRIN experi- ment with tritium. Eur. Phys. J. C 80, 264 (2020). https://doi.org/10.1140/epjc/s10052-020-7718-z

[2.] Gimsa, A., The beauty of nature, $2^{\text {nd }}$ edition, p.31, p.42, Gieselmann Druck- und Medienhaus, Potsdam, Germany 2014, ISBN 978-3-946410-03-4

[3.] Gimsa, A., Der Wellenwiderstand des Vakuums, Teil 1, S.4, Kuss GmbH Potsdam, Germany, 2015, ISBN 978-3-00-051924-6

[4.] Gimsa, A. The metric of space-time, English-German, $2^{\text {nd }}$ edition, p.32-38, p.42, p.55, p.59, p.8385 published by the autor, Germany, 2020, ISBN 978-3-00-064784-0

[5.] P. S. Laplace, A Treatise in Celestial Mechanics, translated by N. Bowditch. Chelsea / New York 1966 Volume IV, Chapter VII 